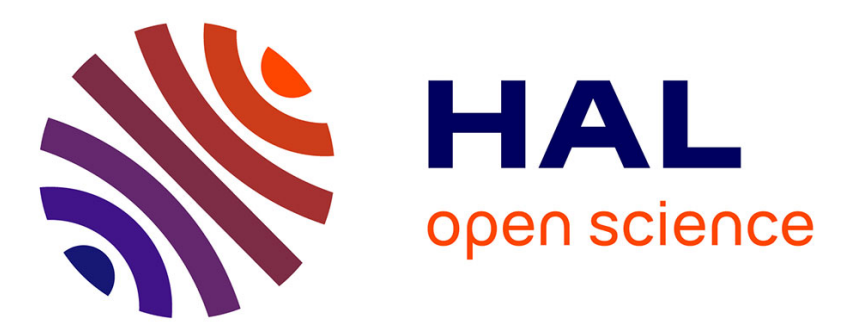

\title{
In-silico Phantom Axon: Emulation of an Action Potential Propagating Along Artificial Nerve Fiber
}

Olivier Rossel, Fabien Soulier, Serge Bernard, David Guiraud, Guy Cathébras

\section{To cite this version:}

Olivier Rossel, Fabien Soulier, Serge Bernard, David Guiraud, Guy Cathébras. In-silico Phantom Axon: Emulation of an Action Potential Propagating Along Artificial Nerve Fiber. ISVLSI: International Symposium on Very Large Scale Integration, Jul 2015, Montpellier, France. pp.228-230, 10.1109/ISVLSI.2015.123 . lirmm-01237977

\section{HAL Id: lirmm-01237977 https://hal-lirmm.ccsd.cnrs.fr/lirmm-01237977}

Submitted on 4 Dec 2015

HAL is a multi-disciplinary open access archive for the deposit and dissemination of scientific research documents, whether they are published or not. The documents may come from teaching and research institutions in France or abroad, or from public or private research centers.
L'archive ouverte pluridisciplinaire HAL, est destinée au dépôt et à la diffusion de documents scientifiques de niveau recherche, publiés ou non, émanant des établissements d'enseignement et de recherche français ou étrangers, des laboratoires publics ou privés. 


\title{
In-silico Phantom Axon: Emulation of an Action Potential Propagating Along Artificial Nerve Fiber
}

\author{
Olivier Rossel $^{*}$, Fabien Soulier $^{\dagger}$, Serge Bernard ${ }^{\ddagger}$, David Guiraud $^{*}$, Guy Cathébras ${ }^{\dagger}$ \\ ${ }^{*}$ INRIA/DEMAR, LIRMM, Montpellier, France \\ †Université Montpellier, LIRMM, Montpellier, France \\ ${ }^{\ddagger}$ CNRS, LIRMM, Montpellier, France \\ $\{1-4\} @$ lirmm.fr
}

\begin{abstract}
This paper presents an original method to emulate a single fiber action potential in a quasi-infinite conductive volume, suitable for reproducible testing of bio-potential recording systems. The model is accurate for reproducing the biopotential even for a small electrode to fiber radial distances. Established current activities of axon is used and programmed in the developed FPGA-based instrument, the model takes into consideration action potential propagation properties, electrode to fiber radial distances, medium conductivity. This paper investigates differences in the action potential amplitude for two longitudinal probe positions one in front of a node of Ranvier (NOR) and one between two NOR, for a large range of radial distances. Results are reported and compared with simulation with a correlation level of $97.6 \%$. The model is realistic enough to help the design of new recording systems.
\end{abstract}

\section{INTRODUCTION}

ElectroNeuroGram (ENG) recordings were investigated for years in order to provide useful data to control neuroprosthetic devices [1] [2] [3] For chronic applications, signals can be acquired through cuff electrodes with various geometries.The number and location of contacts become critical to get richer signals. The sizes of contacts are becoming smaller, distances between them could be reduced, increasing the spatial discretization.

About cuff electrode design, researches focus on increasing the efficiency of ENG measurements, such as optimizing Signal to Noise Ratio (SNR) [4] [5] [6], increasing selectivity linked to axon location (spatial selective recording SSR) and types of fiber (velocities selective recording VSR). SSR and VSR are mainly achieved through a wide variety of designs dealing with the number and position of contacts [7], [8] and [9] [10] (respectively). Generally the theoretical development are based on single fiber action potential (SFAP) properties and are then generalized to compound action potential (CAP). Each design has to be validated. First, electrode prototypes are designed and tested through numerical models. Then, theoretical benefit of new electrode prototypes are confronted to in-vivo experimental validation. But it exists an intermediate step: the validation through emulation, being increasingly used.

The emulation of nerve property through in-silico experiments presents an intermediate validation step. It avoids the constraints of in-vivo experiments but is closer to the real-case compared to numerical model. It often consists in emulating the recording of one SFAP or CAP [11] [12], or a single

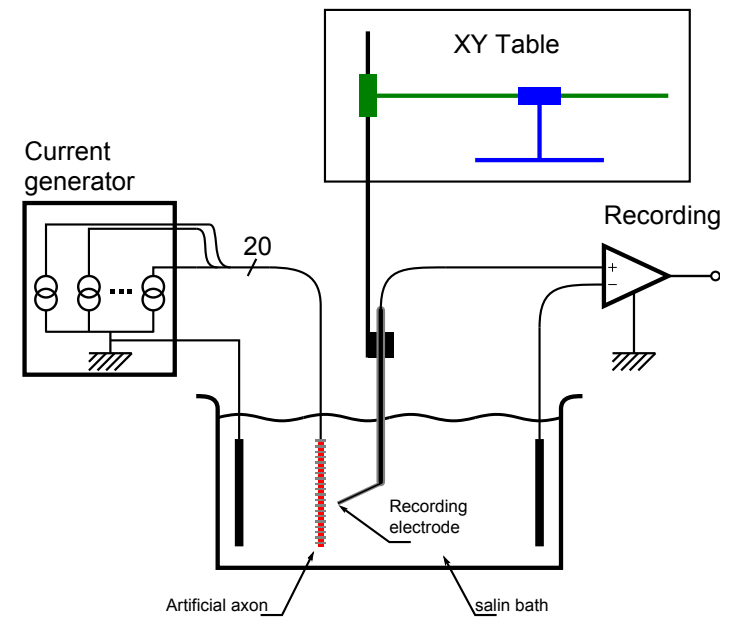

Figure 1. Experiment principle.

NOR [13]. L. Andreassen [13] proposed a method based on this principle to study the spatial transfer function of a cuff electrode. This paper demonstrates that SFAP can be built knowing this transfer function and the current AP on each NOR of an axon. In [11] [12] electrical model of nerve to AP and ENG recording is presented, the model is suitable for conduction velocity, and test different VSR.

The main focus of this work, is to develop a prototype able to emulate an axon fiber at the node of Ranvier (NOR) level allowing SFAP representation.

\section{Method}

We developed a 20 channels programmable and controlled current sources, to mimic ionic currents flowing through 20 NOR of a natural axon. To do so, a simulation of electrical activity of a nerve fiber is computed, the resulting ionic current are programmed in the device. The programmed current flow through 20 contacts disposed in line, placed in a saline bath, it is the phantom axon. Then, measurement of extracellular potential in the saline is carried out using a monopolar probe. A XY table controlled by the computer is used for setting the position of the measuring probe. 


\section{A. Current sources and measurement setup description}

To generate the current on each artificial NOR (Fig. 1), we designed a programmable current generator with 20 independent channels. The desired current is set and programmed on a FPGA (Field Effect Programmable Array, XILINX Nexys 2) that controls 20 12-bit digital-to-analog converters (Analog Device AD7564). These converters produce a differential current. A current mirror based on bipolar transistors is used to provide a bidirectional current (positive or negative) from the differential output of the DAC. These currents are delivered on the contacts of the electrode. It is well known that DC component in the generated currents could lead to rapid deterioration of electrode contacts. We thus added a serial capacitor on each output channel to ensure a null charge balance. To cancel voltage drift, a discharge circuit is added between the output of the current generator and the electrode. At the end of a current generation phase, the discharge circuit switches the capacitor from the current generator to a resistor pulled to the ground voltage. Finally, no charge is accumulated and no voltage drift occurs.

The linearity of the multichannel current generator is limited by the characteristics of the DAC (accuracy of \pm 1 LSB and \pm 4 LSB of gain error) and the current mirror. To increase the accuracy, a digital correction is added to minimize errors and allow full consistency between the 20 channels. The correction relies on a look-up table (LUT) filled up from a calibration phase: first, real output from each code are measured, and then a corrected code is determined. Finally, we designed a highly accurate current generator, with a full scale of $800 \mu \mathrm{A}$ $(-400$ to $+400 \mu \mathrm{A})$, and an absolute error less than $0.4 \mu \mathrm{A}$ (regardless of the digital code and the channel). Thus the relative error lies below $0.05 \%$.

The contact of the monopolar probe is composed of a section of a stainless steel wire with a diameter of $75 \mu \mathrm{m}$, surrounded by an insulating sheath. We perform a differential measurement with a reference electrode formed by a large contact located far enough to consiedr point like measurement.

A Multichannel MPA32I amplifier with a gain of 10 , is used for amplifying the signal. It is then acquired via a Data Acquisition (DAQ) card from National Instruments (NI6259) connected to a computer. The measurement protocol follows these steps: first, a signal generated by the FPGA is used to switch on the current generator and the signal acquisition device. Then, data from the amplifier are recorded by the DAQ. Finally the DAQ generates a signal (Trig - Acquisition) to allow displacement of the probe to a new position.

\section{B. Current configuration}

We used a model of axon to generate simulated values of the transmembrane current as a function of time. We used the study [14] to set realistic parameters for the modeled axon, simulated with the Neuron software (http://www.neuron.yale. edu/neuron/).

We modeled an axon with 100 NOR, set the sample frequency to $400 \mathrm{kHz}$ then we computed $1.28 \mathrm{~ms}$ of axon activity (corresponding to 512 time samples. To ensure a favorable

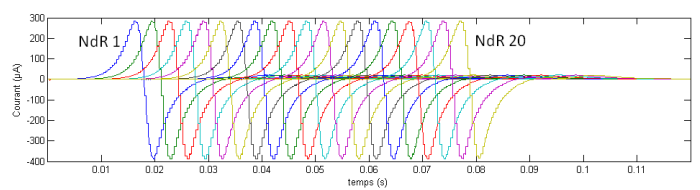

Figure 2. Current delivered by the 20 sources on the 20 contacts of the phantom axon. Only the first 300 time samples are represented, corresponding to the first $0.75 \mathrm{~ms}$ over $1.28 \mathrm{~ms}$ of the simulation.

SNR, the amplitude of nodal currents is set at $400 \mu \mathrm{A}$ ( it is around tens of nanoampere in a natural axon). The 20 central computed transmenbrane currents are saved in a database to be used as inputs to the 20 channels of the current generator. This database corresponds to a $20 * 512$ matrix Fig. 2). The current configuration of the 20 contacts is maintained during the measuring time.

\section{Phantom axon}

To represent an axon of $8.7 \mu \mathrm{m}$ of diameter, with distance between NOR around $1.2 \mathrm{~mm}$, we used an electrode with 20 contacts, disposed in line, the distance between contact being $1.2 \mathrm{~mm}$. Practically we use a cochlear electrode (courtesy from MXM-Neurelec, Vallauris, France). The phantom axon is placed in a saline bath (sodium chloride $0.9 \%$ ), which conductivity is close to in-vivo environment. The monopolar probe is used to measure the electric potential close to the fiber. This probe stays near the middle of the phantom, to avoid end effects. To get an accurate image of the potential around the phantom axon, the position of the measuring probe is controlled and adjusted via a micrometer screw system.

The XY table controlled by the computer (Fig 1) is used for setting the position of the measuring probe. Two longitudinal positions of the probe are set, the first one in front of a NOR and the second one equidistant to two adjacent NOR. 41 radial positions of the probe are set from $250 \mu \mathrm{m}$ to $1000 \mu \mathrm{m}$ (step of $250 \mu \mathrm{m})$.

\section{RESULTS}

The measured signals are substantially identical to those expected. For example the correlation coefficient between measured and simulated potentials for the radial distances of $250 \mu \mathrm{m}$ for two longitudinal positions; in front of a NOR and equidistant to two adjacent NOR (not shown) are respectively $99.8 \%$ and $99.6 \%$. Comparison of the signals is then extended to all the measurement sites the total correlation index amounted to $97.6 \%$.

For this example, it can be noticed that SFAP amplitude recorded in front of the NOR is twice that one measured between two NOR, $(0.6 \mu \mathrm{V}$ Vs $0.3 \mu \mathrm{V}$ reported in Fig. 3 ).

Quantitative results are presented in the Fig. 3. it shows the relationship between SFAP amplitude and electrode to fiber distance. A logarithmic scale representation is used for the radial distances and for the peak-to-peak value of AP . 


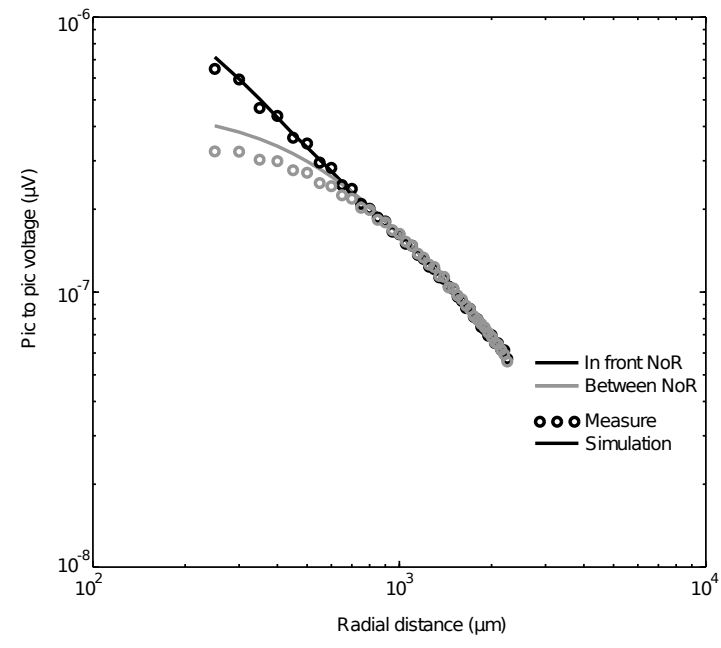

Figure 3. Comparisons between the measured attenuation in front of a NOR and between two NOR.

\section{Discussion}

Concerning the experimental setup and the phantom axon validity, the main difference between the phantom and a real axon is the size of the NOR. But using the duality of the transfer equation, modeling point-small NOR and a probe electrode of length $L$ gives the same results as modeling NOR with a length of $L$ and a point-small probe electrode.

The first contribution is that the measurements performed with the phantom are very close to the simulated potentials. The index of correlation between the two of $97.6 \%$ is very high, proving the relevance of the simulation model and the technique used to emulate an artificial axon. Based on this validation, we demonstrate through both simulation and emulation that: i) the amplitude of the SFAP measured between two NOR is lower than in front of a NOR, for the same radial distance. ii) this effect is only observable at small radial distances, whereas for higher radial distances the difference decreases down to zero. This difference in amplitude of the AP could even be used to give an indirect estimation of the electrode-to-fiber distance.

So, selective and efficient recordings, sensitive to longitudinal positioning should be designed with small contacts and inter distances lower than $1 \mathrm{~mm}$.

\section{CONCLUSiON}

The simulation model together with the emulation were validated and gave consistent results. These results are also consistent with the literature. This setup can be used to evaluate and validate a large span of applications, such as recording device for SSR as well as VSR.

More complex configurations, such the cuff behavior are hard to simulate numerically but can easily be investigated with the phantom setup. As well, further experimental recording designs that can not be validated in true in-vivo environments can be evaluated with the phantom axon.

\section{ACKNOWLEDGEMENT}

This work was supported by Axa research foundation.

\section{REFERENCES}

[1] X. Navarro, T. B. Krueger, N. Lago, S. Micera, T. Stieglitz, and P. Dario, "A critical review of interfaces with the peripheral nervous system for the control of neuroprostheses and hybrid bionic systems," Journal of the Peripheral Nervous System, vol. 10, no. 3, pp. 229-258, Sep. 2005. [Online]. Available: http://doi.org/10.1111/j.1085-9489.2005.10303.x

[2] G. S. Dhillon and K. W. Horch, "Direct neural sensory feedback and control of a prosthetic arm," Neural Systems and Rehabilitation Engineering, IEEE Transactions on, vol. 13, no. 4, pp. 468-472, Dec. 2005. [Online]. Available: http://doi.org/10.1109/TNSRE.2005.856072

[3] P. M. Rossini, S. Micera, A. Benvenuto, J. Carpaneto, G. Cavallo, L. Citi, C. Cipriani, L. Denaro, V. Denaro, G. Di Pino, F. Ferreri, E. Guglielmelli, K.-P. Hoffmann, S. Raspopovic, J. Rigosa, L. Rossini, M. Tombini, and P. Dario, "Double nerve intraneural interface implant on a human amputee for robotic hand control." Clinical neurophysiology : official journal of the International Federation of Clinical Neurophysiology, vol. 121, no. 5, pp. 777-783, May 2010. [Online]. Available: http://doi.org/10.1016/j.clinph.2010.01.001

[4] Z. Yang, Q. Zhao, E. Keefer, and W. Liu, "Noise characterization, modeling, and reduction for in vivo neural recording," in Advances in Neural Information Processing Systems 22, Y. Bengio, D. Schuurmans, J. Lafferty, C. K. I. Williams, and A. Culotta, Eds., 2009, pp. 2160-2168. [Online]. Available: http://books.nips.cc/papers/files/nips22/NIPS2009_ 0025.pdf

[5] E. Huigen, A. Peper, and C. a. Grimbergen, "Investigation into the origin of the noise of surface electrodes." Medical and Biological Engineering and Computing, vol. 40, no. 3, pp. 332-8, May 2002.

[6] X. Liu, A. Demosthenous, and N. Donaldson, "Platinum electrode noise in the ENG spectrum." Medical and Biological Engineering and Computing, vol. 46, no. 10, pp. 997-1003, Oct. 2008. [Online]. Available: http://www.ncbi.nlm.nih.gov/pubmed/18777185

[7] J. Zariffa, M. K. Nagai, Z. J. Daskalakis, and M. R. Popovic, "Influence of the number and location of recording contacts on the selectivity of a nerve cuff electrode," Neural Systems and Rehabilitation Engineering, IEEE Transactions on, vol. 17, no. 5, pp. 420-427, Oct. 2009. [Online]. Available: http://doi.org/10.1109/TNSRE.2009.2023301

[8] O. Rossel, F. Soulier, S. Bernard, and G. Cathébras, "New electrode layout for internal selectivity of nerves," in Engineering in Medicine and Biology Society, 2009. EMBC 2009. Annual International Conference of the IEEE, 2009, pp. 3798-3801. [Online]. Available: http://doi.org/10.1109/IEMBS.2009.5334437

[9] J. Taylor, N. Donaldson, and J. Winter, "Multiple-electrode nerve cuffs for low-velocity and velocity-selective neural recording," Medical and Biological Engineering and Computing, vol. 42, no. 5, pp. 634-643, 2004. [Online]. Available: http://doi.org/10.1007/BF02347545

[10] J. Taylor, M. Schuettler, C. Clarke, and N. Donaldson, "The theory of velocity selective neural recording: a study based on simulation," Medical and Biological Engineering and Computing, vol. 50, no. 3, pp. 309-318, Feb. 2012. [Online]. Available: http://doi.org/10.1007/s11517-012-0874-z

[11] R. Rieger, M. Schuettler, and S.-c. Chuang, "A device for emulating cuff recordings of action potentials propagating along peripheral nerves," vol. 22 , no. 5, pp. 937-945, 2014.

[12] A. Al-Shueli, C. Clarke, N. Donaldson, and J. Taylor, "Improved Signal Processing Methods for Velocity Selective Neural Recording Using Multi-Electrode Cuffs," ... Circuits and Systems, ..., vol. 8, no. 3, pp. 401-410, 2014. [Online]. Available: http://ieeexplore.ieee.org/xpls/abs_ all.jsp?arnumber $=6605584$

[13] L. N. S. Andreasen, J. J. Struijk, and M. K. Haugland, "An artificial nerve fiber for evaluation of nerve cuff electrodes," in Engineering in Medicine and Biology Society, 1997. Proceedings of the 19th Annual International Conference of the IEEE, vol. 5, 1997, pp. 1997-1999 vol.5.

[14] C. C. McIntyre, A. G. Richardson, and W. M. Grill, "Modeling the excitability of mammalian nerve fibers: Influence of afterpotentials on the recovery cycle," Journal of Neurophysiology, vol. 87, no. 2, pp. 995-1006, 2002. [Online]. Available: http://jn.physiology.org/content/ 87/2/995.abstract 